\title{
The Conjecturing Process: Perspectives in Theory and Implications in Practice
}

\author{
María C. Cañadas \\ Universidad de Zaragoza, Spain \\ Jordi Deulofeu \\ Universitat Autònoma de Barcelona, Spain \\ Lourdes Figueiras \\ Universitat Autònoma de Barcelona, Spain \\ David Reid \\ Acadia University, Canada \\ Oleksiy Yevdokimov \\ University of Southern Queensland, Australia
}

\begin{abstract}
In this paper we analyze different types and stages of the conjecturing process. A classification of conjectures is discussed. A variety of problems that could lead to conjectures are considered from the didactical point of view. Results from a number of research studies are used to identify and investigate a number of questions related to the theoretical background of conjecturing as well as practical implications in the learning process.
\end{abstract}

Many researchers have suggested that problem solving and conjecturing are important parts of mathematical activity. Polya (1954), for example, provided the basis for analyzing the conjecturing process in detail by taking into account the roles of specializing and generalizing in mathematical activity. In mathematics education, an important role for conjecturing has also emerged. These days, teaching mathematics through students' active participation in mathematical situations is the goal for many different national mathematics standards. Clearly conjecturing is an important part of an inquiry based approach. Although inquiry based approaches are in the educational spotlight and many research papers

María C. Cañadas is a Profesor Ayudante in the Departamento de Matemáticas at the Universidad de Zaragoza, Spain (mconsu@unizar.es).

Jordi Deulofeu is a titular in the Departamento de Didàctica de la Matemàtica i de les Ciències. Universitat Autònoma de Barcelona, Spain (Jordi.Deulofeu@uab.es).

Lourdes Figueiras is a lectora in the Departamento de Didàctica de la Matemàtica i de les Ciències. Universitat Autònoma de Barcelona, Spain (Lourdes.Figueiras@uab.es).

David Reid is an Associate Professor in the School of Education at Acadia University, Canada (david.reid@acadiau.ca).

Oleksiy Yevdokimov is a Lecturer in the Department of Mathematics and Computing at the University of Southern Queensland, Australia (yevdokim@usq.edu.au). 
appear every year, there is still a need for further theoretical background.

Significant contributions to the topic of conjecturing have been made by many researchers. Fischbein (1987) considered conjectures as expressions of intuitions. Mason (2002) showed the importance of a conjecturing atmosphere. Research has been carried out on the production of conjectures within a dynamic geometry environment (Arzarello et al., 1998; Furinghetti \& Paola, 2003). Recently, Bergqvist (2005) analyzed how students verify conjectures and how teachers' beliefs are related to this process. Amid all this research, however, is a certain lack of clarity about what exactly is being discussed, what exactly is "conjecturing," and how it is related to the most common context for conjecturing suggested in the mathematics education literature: problem solving.

Clearly, problem solving and conjecturing as mathematical activities are intertwined in many ways. However, not all problems lead to conjecturing, and different problems lead to different kinds of conjecturing. As a basis for research into the relationship between problem contexts and conjecturing, we would like to consider a conjecturing process from a theoretical point of view and provide different examples for practical usage. The purpose of this paper is to consider factors in problems that allow or inhibit conjecturing, types of conjecturing, and implications for teaching.

Such an analysis is important to both researchers and teachers in selecting problems for use in classrooms. If the intent is to observe and encourage conjecturing, problems can be selected that allow for conjecturing to occur. If specific types of conjectures are desirable (to create a context for proving, for example) then the choice of problems can encourage, or discourage, certain types. In research, if the suitability of a problem as a teaching context is being analysed empirically, then a rich language for describing students' behaviours is needed.

In this paper, we bring together perspectives and research on conjecturing from Australia, Canada, Spain, and Ukraine to address the following questions:

- What are types and stages of conjecturing?

- What problems can give rise to what types of conjecturing?

- How might we characterize the features of "conjecturing behaviour" of individuals?

Also, we raise other related questions:

- How might teachers be able to teach students to make conjectures?

- Why do the most teachers prefer not to use or promote conjecturing activities in the classroom?

- What are students' and teachers' obstacles in learning and teaching conjecturing?

We offer these questions as topics for further research in mathematics education.

\section{Usage of "Problem"}

We will assume that a problem has two defining characteristics: 
- No solution is immediately available to the solver (in other words, the task is not a trivial exercise, practicing a skill learnt previously).

- It is possible for the solver to find a solution, based on the solver's prior knowledge (AFKS, Yevdokimov, 2003).

This second point is an important condition as it excludes impossible tasks from being considered to be problems. For example, to find the area bounded by two intersecting parabolas is an impossible problem for a six year old because their prior knowledge is inadequate.

As Yevdokimov (2005) points out, problems can be open or closed in various ways. He writes specifically about problems involving properties of geometric figures, but a similar classification can be applied to other types of problems. A problem can be closed: two sets of properties are given and the problem is to prove that one set of properties is a consequence of the other. Or a problem can be open, in three different ways: (a) initial properties can be given and the problem is to find consequences of them, (b) final properties can be given and the problem is to find initial properties of which they are consequences, or (c) no properties at all are given and the problem is to find properties that are related.

Yevdokimov's classification of problems in "open" and "closed" is similar to Polya's (1945) distinction between problems to prove and problems to find. In a problem to prove, the objective is to conclude if an assertion, stated without any ambiguity, is true or false. Such an assertion has two parts: the hypothesis, usually introduced by "if," and the conclusion, usually introduced by "then". One example of such a problem from Euclidean geometry of the plane is the following:

“The sum of the angles inside a triangle is 180 degrees.”

Such a statement could also be formulated by saying:

"IF a polygon is a triangle, THEN the sum of its interior angles is 180 degrees.”

The second type of problems, which are called "problems to find" or construction problems, have the aim of finding a certain object, which satisfies the conditions of the problem by connecting data and unknown. Taking into account this classification, we can demonstrate another example of the same problem:

"If the sum of the interior angles of a geometric figure is 180 degrees, must it be a triangle?”

Or, more generally,

"Does some kind of dependency exist between the number of interior angles of a geometric figure and the total sum of its interior angles? Should some other additional conditions of a geometric figure be taken into consideration?” 
It is worth noting that many problems could be reformulated to appear as either a proof problem or a construction problem. The famous Goldbach conjecture, for example, could be given as a proof problem:

Prove that if $n$ is an even number, $n \geq 4$, then $n$ is the sum of two prime numbers.

The conjecture could also be given as a construction problem (see Problem 1, below). Undoubtedly, construction problems promote rich situations for conjecturing, but other factors (discussed below) are also important.

In the following, we will attempt to relate different types and stages of conjecturing to different characteristics of problems.

\section{The Types, Stages, and Contexts of Conjecturing}

Recall that we are interested in the types and stages of conjecturing and how problems can give rise to conjecturing. From our research and discussions we have characterized five types of conjecturing and identified stages of conjecturing associated with those types. We have also explored how the context of a problem affects the conjecturing that it inspires. In the following three sections, we will describe types, stages and contexts of conjecturing.

\section{Types}

The five types of conjecturing we will describe here are familiar in mathematics education research, but have not, thus far, been systematically compared and considered as types within the larger process of conjecturing. They include empirical induction from a finite number of discrete cases and from dynamic cases, analogy, abduction and perceptually based conjecturing. Each will be described in turn below. We do not claim this list is exhaustive, only that it offers precise descriptions of types of conjecturing often observed.

\section{Type 1: Empirical Induction from a Finite Number of Discrete Cases. A} conjecture can be made based on the observation of a finite number of discrete cases, in which a consistent pattern is observed. This type of conjecture is frequently found in problems involving numbers. In some situations, but not all, the conjecture can be proved by mathematical induction once the general rule has been found $d^{\mathrm{i}}$.

\section{Problem 1}

As a mathematical recreation, one junior high school teacher asked his class if they could find a relationship between the even numbers greater than 4 and the sums of two odd prime numbers. Here are some of the relations that these pupils discovered:

$$
\begin{array}{ll}
6=3+3 & 16=3+13=5+11 \\
8=3+5 & 18=5+13=7+11 \\
10=3+7=5+5 & 20=3+17=7+13
\end{array}
$$




$$
\begin{array}{cc}
12=5+7 & \cdots \cdots \cdots \\
14=3+11=7+7 & 30=7+23=11+19=13+17
\end{array}
$$

After working a while on this problem, one youngster exclaimed, "this could go on forever. There is no end to it.” Smith \& Henderson (1959, p. 123)

The "this" that the child referred to implicitly is Goldbach's conjecture: that any even number greater than 4 can be written as the sum of two odd primes.

It is important to notice the didactical importance of a critical study of cases in this type of conjecturing. Consider, for example, the problem of finding the maximal number of regions in which $n$ intersecting circles divide the plane. Many students, after exploring what occurs for $n=1,2,3$, guess that the general rule is $2^{n}$, which is certainly not the case.

Type 2: Empirical Induction from Dynamic Cases. A conjecture can be made of a general rule that describes the nature of a set of dynamically related events. The basis for the conjecture is an apparently infinite number of continuous events, which are, however, only a subset of the infinite number of possible events accounted for by the conjectured general rule.

For example, in exploring the Black Box activity shown in Figure 1 (The point $\mathrm{X}$ in the triangle is the intersection of the medians, but this is not known when the Black Box activity is done. See Knipping \& Reid, 2005) one might explore by manipulating the triangle $\mathrm{ABC}$ and observing the point $\mathrm{X}$. Through these manipulations one might conjecture that the point $\mathrm{X}$ can never be outside the triangle.

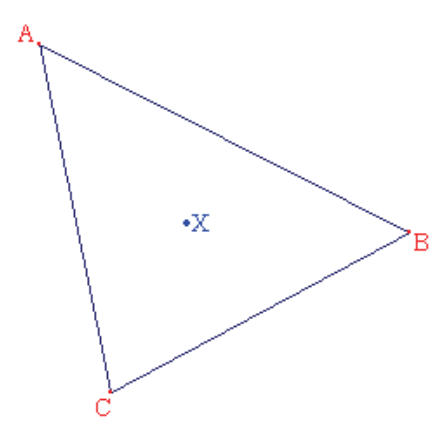

Figure 1: A Black Box problem

Type 3: Analogy. A conjecture can be made by analogy to something already known fact. A general rule can be conjectured on the basis of another known general rule, or a specific fact conjectured on the basis of another known fact.

Problem 2

Given a triangle $\mathrm{ABC}$ and a point $\mathrm{P}$ inside the triangle construct the three lines from each vertex A, B, C to the point P. What can you say about the relationships between the lines and the sides of the triangle? 
For example, given Problem 2, one might think:

"I know that if two lines cut the sides at the midpoint, then the third one will too, because the medians meet in one point. I bet that if two lines cut the sides in a 2:1 ratio, the third one will, too".

Although the conjecture is false in this case, it should not be assumed that such conjectures are not productive. Conjectures made by analogy are very important from a problem solving point of view, as they relate to common strategies, such as reducing the problem to a simpler one, distinguishing cases, and observing symmetries and other geometrical properties.

The importance, or even relevance, of analogy to a discussion of conjecturing from the point of view of mathematics education might be questioned. However it is clear that conjectures involving analogy, and analogy in general, are important in professional mathematics. Polya (1954) pointed this out, and Banach (1932) wrote:

A mathematician is a person who can find analogies between theorems; a better mathematician is one who can see analogies between proofs and the best mathematician can notice analogies between theories; and one can imagine that the ultimate mathematician is one who can see analogies between analogies. (Quoted in Morrison, 2001, p. ix)

Given the centrality of analogy to mathematics, we believe it is important to pay adequate attention to it in research in mathematics education. Of course the role of analogy in mathematics is not the same as the role of analogy in learning mathematics, no more than conjecturing itself plays the same role in these two domains. We will not discuss these differences in detail here, though it is worth remembering that quite often in the mathematics education literature questions are raised concerning the compatibility or appropriate usage of similar concepts described by the same names in the domain of mathematics education on the one hand, and mathematicians' research practice on the other. Though these differences exist, there are also important similarities that lead us to consider analogy as a type of conjecturing here.

Type 4: Abduction. A conjecture can be made of a general rule that would explain an otherwise inexplicable event. This is one of Peirce's meanings of abduction (see Reid, 2003). In this type a general rule is conjectured on the basis of a single case, example or event.

For example, in testing the conjecture made by analogy above-_If two lines cut the sides in a 2:1 ratio, the third one will, too"-one arrives at a situation like that shown in Figure 2. Noting that the third ratio is actually close to 1:4 in the case, a surprising coincidence, one might conjecture that in general "if two lines cut the sides in a 2:1 ratio, then the third one will cut its side in a 1:4 ratio." If this is true in general it makes it less surprising that this occurred in this single case.

The general rule that is conjectured can be a new one (as in the previous example) or it can be chosen from a set of rules known previously. Of course, in both cases, some verification of the rule chosen must follow if it is to be 
considered generally true. For example, given the following problem, students might conjecture that the function is one with which they are familiar.

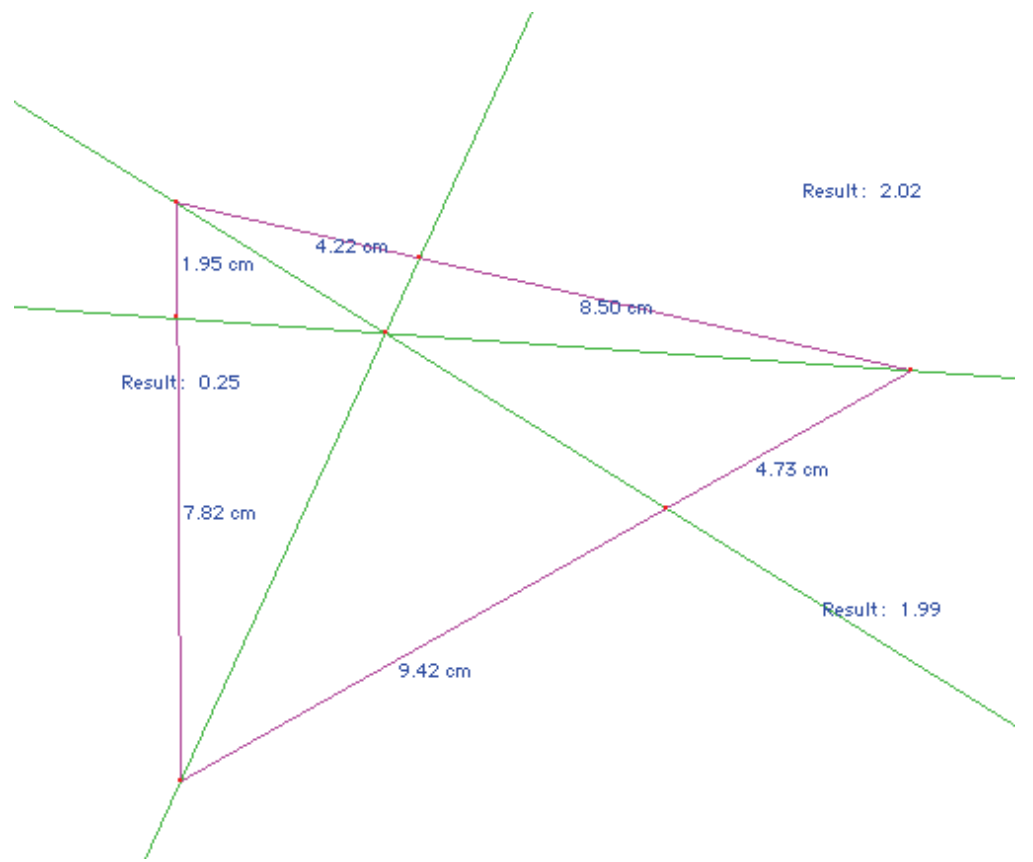

Figure 2: Ratios in triangles

Problem 3

Find the pattern in the following sequence of natural numbers and express it as a function: $3,7,13,21 \ldots$

If the students have worked with arithmetical progressions, they might think of applying the formula for such a progression: $a n=a 1+(n-1) d$ (with an the $n^{\text {th }}$ term and $d$ the difference between two consecutive numbers). However, they will quickly have difficulty settling on a value for $d$ because the given sequence is quadratic. However, their false conjecture might help them to discover that there is a functional relation between the number position and the number of the sequence, which represents an advance in finding the general expression of the pattern.

Type 5: Perceptually Based Conjecturing. A conjecture can be made from a visual representation of a problem or a perceptual translation of its statement. The basis of this type of conjecturing is a careful representation of the content of the problem either concretely or as a mental image. There is not an immediate attention to the relationships existing between the problem's elements because instead the initial focus is on creating a new representation of the problem. Once the representation exists it is often strong enough to accurately reproduce the relationships between the mathematical elements of the problem, and thus to support both conjecturing and proving. De Guzmán (1996) offers the following example. 
Problem 4: The Schroeder-Bernstein problem

Let $\mathrm{A}$ and $\mathrm{B}$ be two sets. Suppose there exists a one-to-one map $f$ from $\mathrm{A}$ into $\mathrm{B}$, and another one-to-one map $g$ from $\mathrm{B}$ into $\mathrm{A}$. Then there exists a bijection $h$ from A into B.

We can make the following representation of the problem: A and B are represented by the two lines and $f$ and $g$ by two descending arrows (see Figure 3 ). Rising arrows represent the inverses $f^{1}$ and $g^{-1}$.

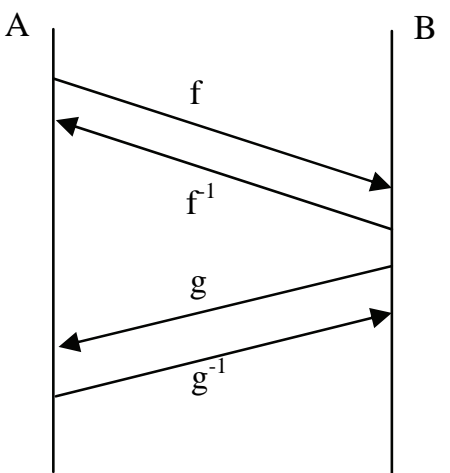

Figure 3: A representation of the Schroeder-Bernstein problem

Now we can consider chains of rising arrows made on these maps, and classify them as follows:

- $\quad$ Class 1: The chain ends on A.

- $\quad$ Class 2: The chain ends on $B$.

- Class 3: The chain has no end, because it has an infinite number of steps, or only one.

This partition also permits us to make a partition of the elements of $\mathrm{A}$ into three disjoint sets, and to make a conjecture of what the bijection $h$ is: If $x$ in A is in a chain of Class 1 , then $h(x)=f(x)$. If $x$ is in a chain of Class 2, then $h(x)=g^{-1}(x)$, and if $x$ is in a chain of Class 3 , then $h(x)=f(x)$. Now the problem remains to prove that $h$ is indeed a bijection, which is the case.

It is clear that the representation made in this example of the sets A and B, and the representation of descending or rising arrows are not the same as the mathematical concepts they represent, but they are clear enough to construct the map $h$ which solves the problem.

This example illustrates that perceptual experience can be an important component in the stimulation of conjecturing.

\section{Stages}

For analysis of conjecturing it is useful to divide the process into stages. Empirical observations of conjecturing suggest that the process occurs in stages, some of which necessarily occur before others. This has been done in some detail for Type 1 conjecturing in previously published research papers. In this section 
we will review the stages of Type 1 conjecturing and then suggest related stages for other types of conjecturing.

Stages in Type 1 Conjecturing. Reid (2001) describes Type 1 conjecturing in terms of several stages:

- Observing a pattern,

- Conjecturing (with doubt) that this pattern applies generally,

- $\quad$ Testing of the conjecture (by predicting a further value and verifying),

- Generalizing of the conjecture (that is, becoming certain of its generality).

Cañadas (2002) and Cañadas \& Castro (2005) offer a more detailed seven stage categorization for describing Type 1 conjecturing:

- Observing cases,

- Organizing cases,

- Searching for and predicting patterns,

- Formulating a conjecture,

- Validating the conjecture,

- Generalizing the conjecture,

- Justifying the generalization.

When observing particular cases the starting point is experiences with particular cases of the problem posed. For the Goldbach conjecture example given above (Problem 1), the stage of observing particular cases might be limited to observing one or two cases: $8=3+5 ; 18=7+11$.

Organizing particular cases involves the use of strategies to systematize and facilitate work with particular cases. The most common strategy used is the organization of particular cases by data lists or tables (Allen, 2001). The students' listing of their sums in increasing order is this stage of the Goldbach conjecture example.

Searching for and predicting patterns is similar to Reid's "pattern observing" but it goes beyond simply observing a pattern. When one observes a repeated and regular situation one naturally imagines that the pattern might apply to the next unknown cases well. Note that this is different from conjecturing that it will apply for all cases, as only the next case is considered. In the Goldbach example, the pattern observed is "All of these even numbers can be expressed as the sum of two prime numbers" and a prediction would be "32 can also be expressed as the sum of two prime numbers."

Formulating a conjecture means making a statement about all possible cases, based on empirical facts, but with an element of doubt. A conjecture is a statement that has not been validated. Saying "Maybe all even numbers can be expressed as the sum of two prime numbers" is formulating a conjecture for the Goldbach example.

Validating the conjecture, like Reid's "testing," involves both making a prediction and verifying the correctness of that prediction by some independent method. This establishes the truth of the conjecture for a new specific case but 
not in general. For the Goldbach example, the prediction that " 32 can also be expressed as the sum of two prime numbers" can be checked by looking for two prime numbers (for example, $3+29$ ) whose sum is 32 .

Generalizing the conjecture involves a change in what Duval (1990) calls its "epistemic value", from a possible conjecture to an accepted general rule. This is a change in what is believed about the statement. If one believes (as the child in the example did) that the Goldbach conjecture is true in general, then one has generalized it. If not, it remains a conjecture.

Looking at additional examples is not sufficient to justify a generalization. Justifying the generalized conjecture involves giving reasons that explain the conjecture, perhaps with the intention of convincing another person that the generalization is justified. If it is necessary, one might create a mathematical proof as the justification that guarantees the truth of the conjecture. So far, no one has managed to justify the Goldbach conjecture.

Note that not all of these stages necessarily occur with every conjecture. These stages can also be applied, with some modifications to other types of conjecturing as we will describe in the next four sections.

Stages in Type 2 Conjecturing. Conjecturing by empirical induction from dynamic cases (Type 2) could proceed through these stages:

- Manipulating a situation dynamically through continuity of cases,

- Observing an invariant property in the situation,

- Formulating a conjecture that the property holds in other cases,

- Validating the conjecture,

- Generalizing the conjecture,

- Justifying the generalization.

Note that the stages at the beginning of the conjecturing process are specific to this type of conjecturing, but those at the end of the process fit into the same pattern as in Type 1 conjecturing.

For example, in the Black Box activity (see Figure 1) the stage of manipulating a situation dynamically through continuity of cases is the manipulation of the triangle $\mathrm{ABC}$ and observation of the point $\mathrm{X}$ and its relationship to the triangle. When one observes that the point $X$ does not move outside the triangle during these manipulations, the second stage occurs. Once the conjecture that the point $\mathrm{X}$ can never be outside the triangle is made, further manipulations occur, focused on validating the conjecture. In contrast to the earlier manipulations, these are focused on trying to move $\mathrm{X}$ outside the triangle, and so extreme cases might be considered that did not initially come to mind as possibilities. When the point $\mathrm{X}$ remains inside the triangle, even in these extreme cases, the conjecture might be generalized. Justifying generalizations in Black Box contexts is generally not possible, however, as the basis for deducing that the point cannot be outside the triangle (the fact that it is the intersection of the medians) is precisely what is hidden in the Black Box. One might make a further conjecture, that $\mathrm{X}$ is the intersection of the medians, which would then justify the generalization of the initial conjecture. Such a conjecture would be of Type 4 
(abduction) as the general rule being hypothesized accounts for an otherwise inexplicable occurrence.

Stages in Type 3 Conjecturing. Conjecturing by analogy (Type 3) could proceed through these stages:

- Observing two cases

- $\quad$ Searching for similarities between the cases

- Formulating a conjecture based on the similarity

- Validating the conjecture

- Generalizing the conjecture

- Justifying the generalization

Recall the example given above:

"I know that if two lines cut the sides at the midpoint, then the third one will too, because the medians meet in one point. I bet that if two lines cut the sides in a 2:1 ratio, the third one will, too."

The two cases are that of the medians of a triangle and that of lines cutting two sides in a 2:1 ratio. These cases have several known similarities: lines pass through vertices and opposite sides of a triangle, the opposite side is cut into a specified ratio, the lines meet, etc. In the case of the medians, another feature is known: all three medians meet in a single point, or, put another way, the line from the third vertex, passing through the intersections of the medians from the first two vertices, is also a median. This feature can be used as the basis for formulating a conjecture, that in the case of lines cutting two sides in a 2:1 ratio, the line from the third vertex, passing through the intersections of the lines from the first two vertices, also cuts the opposite side in a 2:1 ratio. As in the other types of conjecturing, this conjecture would then be validated, generalized and justified, but in this specific example this is not possible as the conjecture is not true. Instead, in this example, the refutation of the conjecture creates a surprising situation, which then leads to Type 4 conjecturing.

Stages in Type 4 Conjecturing. Conjecturing by abduction (Type 4) could proceed through these stages:

- Observing one case

- Observing a surprising or significant feature of that case

- Formulating a conjecture that the feature applies to other cases

- Validating the conjecture

- Generalizing the conjecture

- Justifying the generalization

In the example given above (Figure 2) the figure itself is the case that is observed. The ratio 1:4 is its surprising feature, and in a dynamic geometry context one could immediately validate the conjecture that in general "if two lines cut the sides in a 2:1 ratio, then the third one will cut its side in a 1:4 ratio" by dragging the figure. Once the conjecture is validated it can be generalized, and 
then traditional methods of geometric proof can be used to justify the generalization.

Stages in Type 5 Conjecturing. Perception based conjecturing (Type 5) could proceed through these stages:

- $\quad$ Translating the problem into a perceptual representation

- Constructing a personal mental representation of the mathematical elements involved

- Perceptually observing special features of the representation

- Formulating a conjecture based on the special features of the representation

- Justifying or formalizing the translation

- Generalizing the conjecture

- Justifying the generalization

The step of justifying or formalizing the translation replaces, in this type of conjecturing, the empirical validation of the conjecture present in the other types. What is necessary here is to show that the special features of the representation are in fact directly related to analogous special features in the problem itself. In the Schroeder-Bernstein problem (Problem 4, above) the rising chains of arrows and their classification can be seen in the imagination, supported by the diagram. However, some work is needed to show that these features of the representation correspond to features in the mathematical situation of the two sets. In doing this work the translation to the representation is justified and once that is done the conjecture can be generalized. It remains to justify the generalized conjecture within the original problem context.

\section{Context and representation systems}

It is important to think about the context in which the problem is proposed as different contexts can lead to different types of conjectures. In problem contexts, we can distinguish among different representation systems. What differs from one representation system to another are the mathematical rules which guide the way of working with the particular concept (Goldin \& Shteingold, 2001, pp. 3-4).

Several problems that are identical in their mathematical structure can be posed in very different contexts. For example, consider the following problem:

Problem 5a: The Handshake problem

$n$ people met in a celebration and each shakes hands with all the others.

How many handshakes occurred at the celebration?

Usually, this problem is easier for students to solve if it is posed in the following terms:

Problem 5b: The Diagonals problem 
Given a polygon with $n$ vertices, how many diagonals (lines joining two vertices) can be drawn?

Problem 5a is usually translated into an arithmetic/algebraic form so that it can be approached mathematically. Problem $5 b$ is already expressed in mathematical language so no translation is needed ${ }^{\mathrm{ii}}$. These two forms can lead to different conjectures: the arithmetic/algebraic form giving rise to a Type 1 conjecture and the geometric form giving rise to a Type 5 conjecture.

Let us consider another problem whose context can be expressed in two different ways:

Problem 6

Some natural numbers can be expressed as the sum of two or more consecutive numbers. For example $7=3+4 ; 10=1+2+3+4,12=2$ $+4+5$, etc. Which numbers can be expressed in this way?

Students who try to find a general rule by thinking about particular cases usually find it hard to make a good conjecture. Many suggest "all odd numbers” or "all multiples of three."

If the problem is translated into a visual representation, however, it becomes possible to see the sum of two or more consecutive numbers as a "stair number", which can be decomposed into two parts, a triangular number on top of a rectangular number. This leads to a rule such as $\left(n^{2}+n \div 2\right)+k n$. This tells the students something about the construction of such numbers, but it still does not let them to know if a given number is able to be expressed as required.

More work needs to be done on the relationship between problem contexts and types of conjecturing. The examples we have offered here are intended as models for such work. In the next section we will offer another example, in which types and stages of conjecturing are used to analyze the responses of students to a problem.

\section{Problems and Conjectures: Implications for Teaching}

One problem can give rise to many conjectures, not all of the same type. For example consider the following:

Problem 7: Heron’s problem

Let $s$ be a line and A and B two points on the same side of $s$. For which point $\mathrm{P}$ on $s$ is $\mathrm{AP}+\mathrm{PB}$ the shortest path joining $\mathrm{A}$ and $\mathrm{B}$ ?

Possible conjectures include:

- Conjecture 1: Let $\mathrm{C}$ be the point symmetric to $\mathrm{A}$ with respect to the line $\mathrm{s}$, so that the segment $\mathrm{AC}$ is perpendicular to $\mathrm{s}$. The line joining $\mathrm{B}$ and $C$ intersects $\mathrm{S}$ at $\mathrm{P}$, which is the point we are looking for (see Figure 4, from Figueiras \& Deulofeu, 2005).

- Conjecture 2: $\mathrm{P}$ is the intersection between $s$ and the perpendicular bisector of $\mathrm{AB}$ (see Figure 5). 
- Conjecture 3: $\mathrm{P}$ is the intersection between $s$ and its perpendicular through the mid-point of AB. (see Figure 6).

- Conjecture 4: $r$ is the perpendicular to $\mathrm{BD}$ through $\mathrm{A}$; $\mathrm{O}$ the intersection of $r$ and $\mathrm{BE}$; and $\mathrm{P}$ the intersection between $s$ and its perpendicular through $\mathrm{O}$ (see Figure 7).

- Conjecture 5: $\mathrm{O}$ is the intersection between $\mathrm{AD}$ and $\mathrm{BE} . \mathrm{P}$ is the intersection between $s$ and its perpendicular through $\mathrm{O}$ (see Figure 8).

- Conjecture 6: $\mathrm{P}$ is the point found empirically by moving the point along DE until the measured distance is minimal.

The first conjecture seems not to fit the types proposed earlier, suggesting a more detailed analysis (like that begun in Figueiras \& Deulofeu, 2005) is needed. Conjectures 2 to 5 are related (as will be discussed below), and involve abductions. The final conjecture is of Type 2: Empirical induction from dynamic cases.

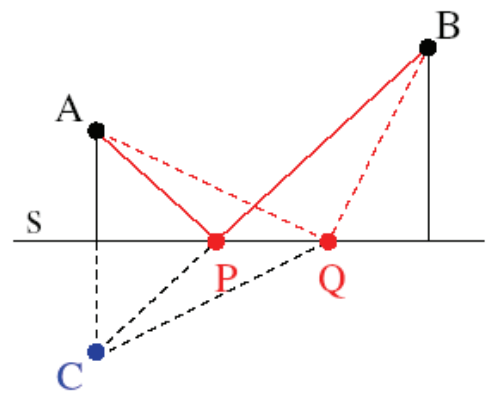

Figure 4: Conjecture 1

Figure 5: Conjecture 2
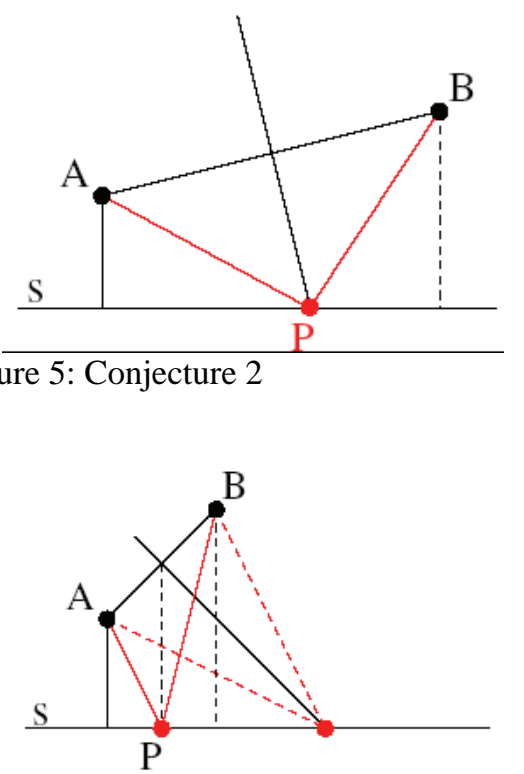

Figure 6: Conjecture 3

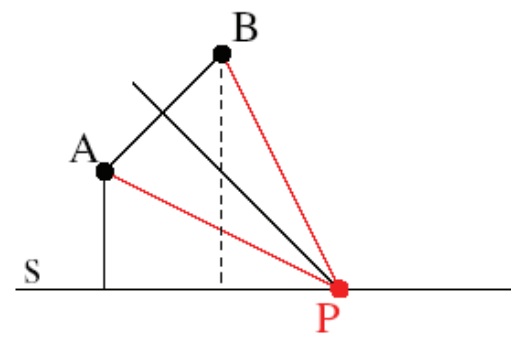

Figure 5a: Counterexample to conjecture 2

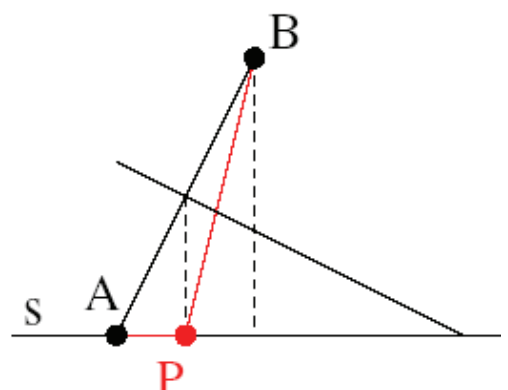

Figure 6a: Counterexample to conjecture 3 


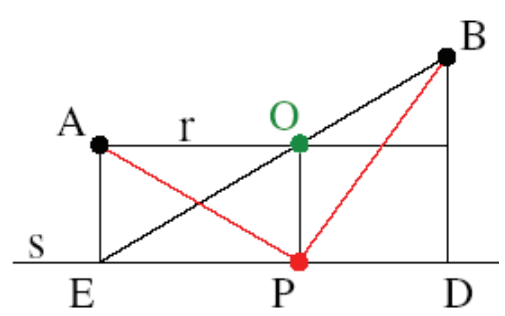

Figure 7: Conjecture 4

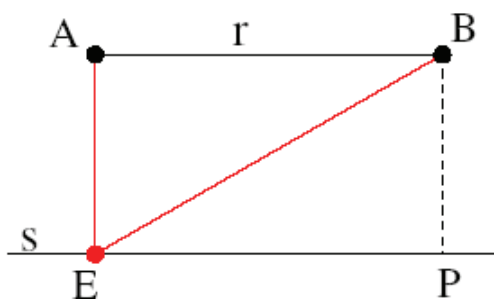

Figure 7a: Counterexample to conjecture 4

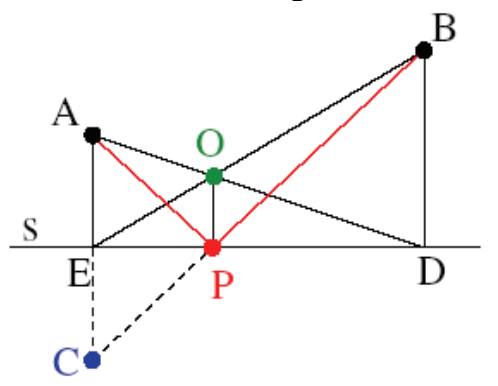

Figure 8: Conjecture 5

The task is to find a shortest distance. What is the Active Fund of Knowledge of the Student (AFKS) in this area of mathematics? AFKS includes the student's understanding of definitions and properties for the mathematical objects of the domain and the skills to use that knowledge. In other words, what do the students actively know about distances?

From their conjectures it seems they know that the shortest distance between two points is a straight line, and also that the perpendicular bisector is the locus of equidistance between two points. Those who made Conjecture 1 had the insight to apply their knowledge that the shortest distance between two points is a straight line, but to do so is not trivial, as other knowledge, related to the preservation of distance under reflection, must come into play.

For conjectures 2-5, the special properties of each diagram influence the conjecturing process. A solution is sought that looks right for the specific diagram at hand.

Consider the diagram shown in Figure 4a. In this special case the position of $\mathrm{P}$ is in the centre, which suggests Conjecture 2: $\mathrm{P}$ is the intersection between $s$ and the perpendicular bisector of $\mathrm{AB}$ (see Figure 5). It is possible that those who made conjecture 2 used their knowledge that the perpendicular bisector is the locus of equidistance between two points. Why this particular knowledge was active for these students is not clear. Perhaps they had solved a problem in which it was important recently? We are aware that some questions need further clarification. At the same time it is reasonable to make use of this knowledge. They are considering a path made up of two parts AP and PB. Not knowing how to minimize the total distance they might have considered the easier problem of minimizing the longer part. If the total distance were fixed then the longer part is minimal when the two arts are equal, which occurs when $\mathrm{P}$ lies on the perpendicular bisector of AB (see Figure 5). Unfortunately, the total distance is not fixed (as we are trying to minimize it) so the reasoning is flawed and a counter example can be found, when the horizontal distance between A and B is 
small compared to the vertical distance between them (see Figure 6). Conjectures 2 and 3 illustrate an interesting confusion that occurs in solving such problems: equal distances are often confused with shortest distances.

This diagram (Figure 6) in the context of the reasoning that has gone before, suggests the next conjecture (Conjecture 3). The problem it reveals is that the point $\mathrm{P}$ should stay on the segment ED that is the projection of AB onto $s$. This constraint, combined with the earlier consideration of trying to equalize distances, suggests that the midpoint of ED, or alternately the projection of the midpoint of $\mathrm{AB}$ onto $s$, is the point $\mathrm{P}$. This new construction also accounts for the known special case shown in Figure 5a. If $\mathrm{A}$ is on $s$, however, a new counterexample is produced (Figure 6a). This diagram shows clearly the correct solution in another specific case: When $\mathrm{A}$ is on $s$ then the shortest path is $\mathrm{AB}$ itself. The emphasis shifts to defining $P$ is such a way that it can be seen as a continuous transformation from this initial situation. As A moves up, P must move to the right. The segment EB provides a mechanism to produce this motion: $\mathrm{A}$ is projected horizontally onto $\mathrm{EB}$ to the point $\mathrm{O}$ and then $\mathrm{O}$ is projected vertically onto $s$ to the point $\mathrm{P}$ (Figure 7). Again a counter-example is not hard to find (Figure 7a), because this construction does not work in the original special case, when EA $=$ DB.

Combining the two special cases (and the mirror image of the second) produces the diagram shown in Figure 8a, which immediately suggests Conjecture 5: $\mathrm{O}$ is the intersection between $\mathrm{AD}$ and $\mathrm{BE}$. $\mathrm{P}$ is the intersection between $s$ and its perpendicular through $\mathrm{O}$ (see Figure 8).

Thus through a sequence of conjectures, each one based on a single special case (except the last which is based on two special cases), a conjecture is produced that turns out to be correct. In this case the conjecturing process does not produce an instant proof (as with Conjecture 1) but neither is completely separated from the proving process (as with Conjecture 6). It provides a few clues for the proving process.

\section{Closing Remarks}

Undoubtedly, conjecturing plays a significant role in an inquiry based learning process. Educational standards in many parts of the world promote inquiry approaches in which students solve problems, make conjectures and justify their conclusions. However, such an approach presents teachers with many challenges. Among these is the need to select problems that can are likely to give rise to conjecturing, and then to guide students' conjecturing in ways that lead to mathematical justifications. This selection process cannot be left to trial and error, and yet there is no generally recognised systematic structure in which to analyze problems for their conjecturing potential and to describe students' conjecturing in terms of its progress towards mathematical justifications. In this article we have attempted to begin filling this gap in the theoretical background of research and teaching focused on conjecturing.

\section{References}

Allen, L. G. (2001). Teaching mathematical induction: An alternative approach. Mathematics Teacher, 94, 500-504.

Arzarello, F., Gallino, G., Micheletti, C., Olivero, F., Paola, D., \& Robutti, O. (1998). Dragging in 
Cabri and modalities of transition from conjectures to proofs in geometry. In A. Olivier and K. Newstead (Eds.), Proceedings of the Twenty-second Annual Conference of the International Group for the Psychology of Mathematics Education (Vol. 2, pp. 32-39). Stellenbosch, South Africa.

Bergqvist, T. (2005). How students verify conjectures: Teachers' expectations. Journal of Mathematics Teacher Education, 8, 171-191.

Cañadas, M. C. (2002). Razonamiento inductivo puesto de manifiesto por alumnos de secundaria. Granada: Universidad de Granada.

Cañadas, M. C. \& Castro, E. (2005). A proposal of categorisation for analysing inductive reasoning. In M. Bosch (Ed.), Proceedings of the CERME 4 International Conference (pp. 401-408). Sant Feliu de Guíxols, Spain. Published online at http://ermeweb.free.fr/CERME4/

De Guzmán, M. (1996). El rincón de la pizarra. Ensayos de visualización en análisis matemático. Pirámide, Madrid.

Duval, R. (1990). Pour une approche cognitive de l'argumentation. Annales de Didactique et de Sciences Cognitives, 3, 195-221.

Figueiras, L. \& Deulofeu, J. (2005). Visualising and conjecturing solutions for Heron's problem. In M. Bosch (Ed.), Proceedings of the CERME 4 International Conference (pp. 420-427). Sant Feliu de Guíxols, Spain. Published online at http://ermeweb.free.fr/CERME4/

Fischbein, E. (1987). Intuition in science and mathematics. An educational approach. Dordrecht: Reidel.

Furinghetti, F. \& Paola, D. (2003). To produce conjectures and to prove them within a dynamic geometry environment: A case study. In N. A. Pateman, B. J. Doherty, \& J. Zilliox (Eds.), Proceedings of the Twenty-seventh Annual Conference of the International Group for the Psychology of Mathematics Education (Vol. 2, pp. 397-404). Honolulu, USA.

Goldin, G. A. \& Shteingold, N. (2001). Systems of representations and the development of mathematical concepts. In A. Cuoco \& F. R. Curcio (Eds.), The Roles of Representation in School Mathematics. National Council of Teachers of Mathematics 2001 Yearbook (pp. 1-23). Reston, VA: National Council of Teachers of Mathematics.

Knipping, C. \& Reid, D. (2005). Schwarze Kisten - Mit Black boxes Zusammenhänge erkunden. In B. Barzel, S. Hußmann, \& T. Leuders (Eds.), Computer, Internet \& Co. im Mathematikunterricht (pp. 167-180). Berlin: Cornelsen.

Mason, J. (2002). Generalisation and algebra: Exploiting children’s powers. In L. Haggerty (Ed.), Aspects of teaching secondary mathematics: Perspectives on practice (pp. 105-120). London: RoutledgeFalmer.

Morrison, T. J. (2001). Functional analysis: An introduction to Banach space theory. New York: Wiley.

Polya, G. (1945). How to solve it: A new aspect of mathematical method. Princeton, NJ: Princeton University Press.

Polya, G. (1954). Mathematics and plausible reasoning. Princeton, NJ: Princeton University Press.

Reid, D. A. (2001). Conjectures and refutations in Grade 5 Mathematics. Journal for Research in Mathematics Education, 33(1) 5-29.

Reid, D. A. (2003). Forms and uses of abduction. In M. A. Mariotti (Ed.), Proceedings of the CERME 3 international conference (n.p.). Bellaria, Italy. Published online at http://ermeweb.free.fr/CERME3/Groups/TG4/TG4_Reid_cerme3.pdf

Smith, E. P. \& Henderson, K. B. (1959). Proof. In Fawcett H., Hach A., Junge C., Syer H., van Engen H., Jones P (Eds.), The growth of mathematical ideas K-12. Twenty-fourth yearbook (pp.111-181). Washington, DC: National Council of Teachers of Mathematics.

Yevdokimov, O. (2003). Intuitive proofs as a tool for development of student's creative abilities while solving and proving. Short Oral presentation. In N. A. Pateman, B. J. Doherty, \& J. Zilliox (Eds.), Proceedings of the Twenty-seventh Annual Conference of the International Group for the Psychology of Mathematics Education (Vol. 1, p. 264). Honolulu, USA.

Yevdokimov, O. (2005). About a constructivist approach for stimulating students' thinking to produce conjectures and their proving in active learning of geometry. In M. Bosch (Ed.), Proceedings of the CERME 4 International Conference (pp. 469-480). Sant Feliu de Guíxols, Spain. Published online at http://ermeweb.free.fr/CERME4/ 


\footnotetext{
${ }^{\mathrm{i}}$ Even in the case of natural numbers there are some properties that can not be proved by mathematical induction, for example, divisibility properties.

${ }^{\text {ii }}$ We do not wish to claim that a problem expressed in mathematical language is necessarily easier to solve than if it is expressed in ordinary language. This is not always the case.
} 